\title{
Frequency Response of Electrolyte-gated Graphene Electrodes and Transistors
}

\author{
Simon Drieschner ${ }^{1}$, Anton Guimerà ${ }^{2,3}$, Ramon G. Cortadella ${ }^{4}$, \\ Damià Viana $^{4}$, Evangelos Makrygiannis ${ }^{1}$, Benno M. Blaschke ${ }^{1}$, \\ Josua Vieten ${ }^{1}$, and Jose A. Garrido ${ }^{4,5}$ \\ ${ }^{1}$ Walter Schottky Institut und Physik-Department, Technische Universität München, \\ Am Coulombwall 4, 85748 Garching, Germany \\ ${ }^{2}$ Institut de Microelectrònica de Barcelona IMB-CNM (CSIC), Campus U.A.B, 08193 \\ Bellaterra (Barcelona), Spain \\ ${ }^{3}$ Networking Center on Bioengineering, Biomaterials and Nanomedicine \\ (CIBER-BBN), Zaragoza, Spain \\ ${ }^{4}$ Catalan Institute of Nanoscience and Nanotechnology (ICN2), CSIC and The \\ Barcelona Institute of Science and Technology, Campus UAB, 08193 Bellaterra, Spain \\ ${ }^{5}$ ICREA, Pg. Llus Companys 23, 08010 Barcelona, Spain \\ E-mail: joseantonio.garrido@icn2.cat
}

\begin{abstract}
.
The interface between graphene and aqueous electrolytes is of high importance for applications of graphene in the field of biosensors and bioelectronics. The graphene/electrolyte interface is governed by the low density of states of graphene that limits the capacitance near the Dirac point in graphene and the sheet resistance. While several reports have focused on studying the capacitance of graphene as a function of the gate voltage, the frequency response of graphene electrodes and electrolytegated transistors has not been discussed so far. Here, we report on the impedance characterization of single layer graphene electrodes and transistors showing that due to the relatively high sheet resistance of graphene, the frequency response is governed by a distribution of resistive and capacitive circuit elements along the graphene/electrolyte interface. Based on an analytical solution for the impedance of the distributed circuit elements, we model the graphene/electrolyte interface both for the electrode and the transistor configurations. Using this model, we can extract relevant material and device parameters such as the voltage-dependent intrinsic sheet and series resistances as well as the interfacial capacitance. The model also provides information about the frequency threshold of electrolyte-gated graphene transistors above which the device exhibits a non-resistive response, offering important insight into the suitable frequency range of operation of electrolyte-gated graphene devices.
\end{abstract}

Keywords: graphene, distributed elements, impedance, electrode, transistor 


\section{Introduction}

Since its first isolation in 2004[1], graphene has attracted significant scientific interest because of its unique properties $[2,3,4,5,6]$ and many potential applications. Many of these applications such as solution-gated field-effect transistors[7], biosensors[8], electrodes[9], and supercapacitors[10, 11] depend on the interface between graphene and aqueous electrolytes, especially around the Dirac point where the highest mobility of charge carriers is observed[3]. However, the density of states near the Dirac point is strongly limited for graphene[12], which explains the low quantum capacitance of this material compared to metals or semiconductors. Therefore, in order to understand the graphene/electrolyte interface, the contribution of the quantum capacitance has to be properly taken into account[13]. Considering the series arrangement of the electrolyte double layer capacitance and the graphene quantum capacitance, the inverse of the total capacitance is the sum of the inverses of the quantum capacitance and the double layer capacitance[14]. Thus, the total capacitance is limited by the capacitor with the smallest value, which is the quantum capacitance near the Dirac point. Many reports have recently focused on studying the total interfacial capacitance and the quantum capacitance in graphene in various electrolytes like ionic liquids[13], ion-gels[15], and aqueous electrolytes[16, 17, 18] using electrochemical impedance spectroscopy $[13,15,16,17,18]$. They show the voltage dependence of the interfacial capacitance in graphene, with a measured capacitance near the Dirac point ranging from $2 \mu \mathrm{F} \mathrm{cm}^{-2}[16]$ to $5 \mu \mathrm{F} \mathrm{cm}^{-2}[13]$. However, these reports only considered experiments at low frequency, thus failing to display the complexity of the graphene/electrolyte interface. Here, we discuss the frequency dependence of the complex impedance of single layer graphene electrodes and transistors as a function of the potential applied to the electrolyte. Our experiments reveal that the frequency response of devices based on the graphene/electrolyte interface is governed by a distribution of elements, resistors and capacitors, along the graphene surface resulting from the limited conductivity of graphene. Using both an analytical and a finite element method based solution to study the distributed elements behavior, we successfully model the frequencydependent impedance of electrolyte-gated single-layer graphene field-effect transistors and electrodes. Using this approach, we derive the intrinsic sheet resistance of graphene, the graphene/electrolyte interfacial capacitance, and the series resistance of electrolytegated graphene devices as a function of the applied electrolyte potential. Furthermore, our analysis identifies the threshold frequency that marks the transition between a resistive and non-resistive response along the transistor channel, or in the case of the electrode between a purely capacitive and mixed response (resistive and capacitive distributed elements). It is shown that this threshold strongly depends on the dimensions of the device active area, i.e. the electrode diameter or the transistor gate area, as well as on the value of the interfacial capacitance and the intrinsic resistance of the graphene sheet. We demonstrate that for typical device dimensions in the mm-range, the threshold frequency can be in the lower $\mathrm{kHz}$ range, which becomes of relevance for applications 
based on electrolyte-gated graphene devices.

\section{Results and discussion}

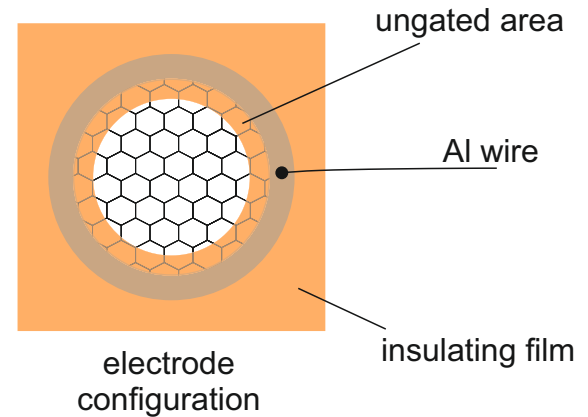

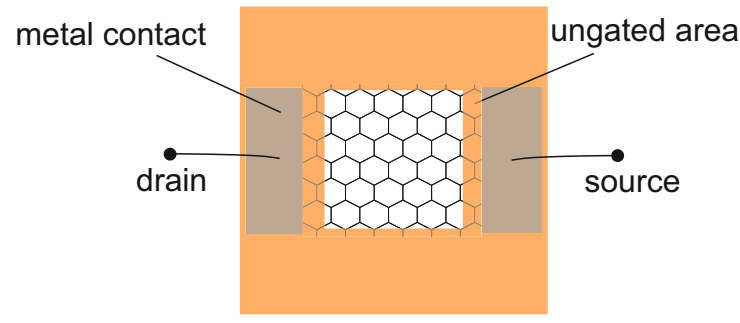

transistor configuration
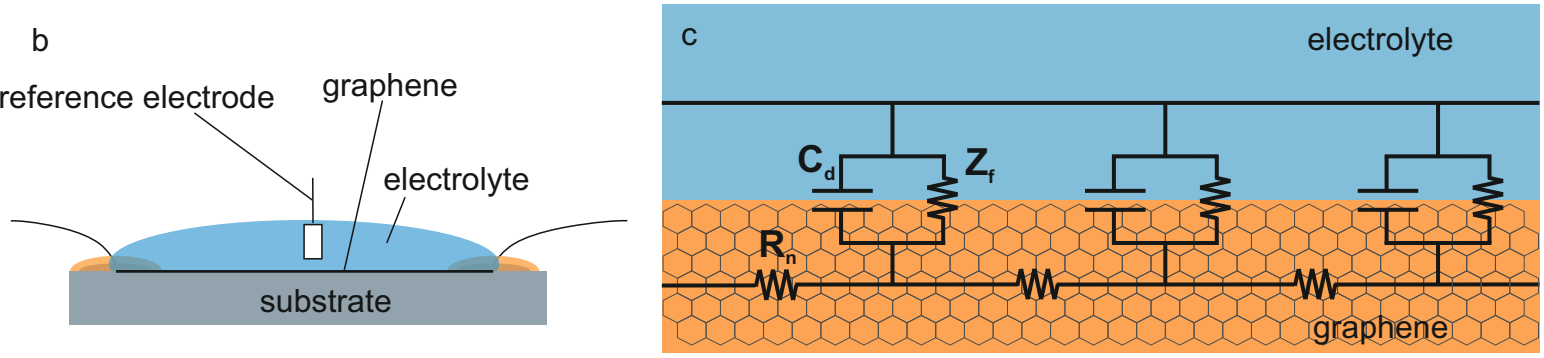

Figure 1. Schematics of the used devices and the electrolyte/interface. (a) Top view of the used configurations: (left) electrode configuration with a circular graphene area, (right) transistor configuration with a rectangular graphene area. (b) Side view of an electrolyte-gated graphene device. The graphene sheet is gated in an aqueous electrolyte using a $\mathrm{Ag} / \mathrm{AgCl}$ reference electrode. (c) Simplified equivalent circuit model at the graphene/electrolyte interface. $R_{n}$ is the sheet resistance, $C_{d}$ represents the interfacial capacitance, and $Z_{f}$ is the Faradaic impedance.

Top-view schematics of the investigated electrode and transistor configurations are depicted in Fig. 1a. Single-layer graphene (SLG) films, as confirmed by Raman spectroscopy (see Supporting Information (S.I.)), were employed in all the experiments in this work. SLG was grown by chemical vapor deposition (CVD) on Cu foils as described elsewhere[19], transferred onto the substrate, and electrically connected using metal contacts. Precise insulation of the contacts with a silicone passivating film reduces the ungated graphene area, i.e. the graphene that is not in contact with the electrolyte, and therefore, the so-called access resistance. In the electrode configuration (Fig. 1a left), a circular area of $0.90 \mathrm{~cm}^{2}$ is exposed to the electrolyte and a circular metal ring is used to minimize the contact resistance. In the transistor configuration, a rectangular area of $0.96 \times 0.96 \mathrm{~cm}^{2}$ was left uncovered and two metal contacts on each side serve as source and drain of the transistor. The potential in the electrolyte is changed using a $\mathrm{Ag} / \mathrm{AgCl}$ reference electrode (see Fig. 1b). More details about the device fabrication can be found in the Methods Section.

The interface between SLG and the electrolyte is illustrated in Fig. 1c. In the 
equivalent circuit model, the interfacial capacitance $C_{d}$ takes into account both the quantum capacitance and the electric double layer capacitance. In order to account for the heterogeneity of electrode/electrolyte interfaces, the capacitance is typically replaced by a constant phase element (CPE)[14]. The Faradaic impedance is represented by $Z_{f}$ and the intrinsic sheet resistance of graphene by $R_{n}[14]$. In the absence of charge transfer induced by Faradaic reactions at the graphene/electrolyte interface, $Z_{f}$ can be removed leading to a distribution of R-C or R-CPE elements along the graphene's surface. In graphene, the values of $R_{n}$ and $C_{d}$ strongly depend on the gate voltage, the applied potential between electrolyte and graphene. As the lowest charge carrier density is observed at potentials near the Dirac point or the so-called "charge neutrality point" [3], a high sheet resistance and a low capacitance can be expected at these potentials. Increasing the gate potential with respect to the Dirac voltage leads to an increase of the charge carrier density and, therefore, to lower $R_{n}$ and higher $C_{d}$ values.
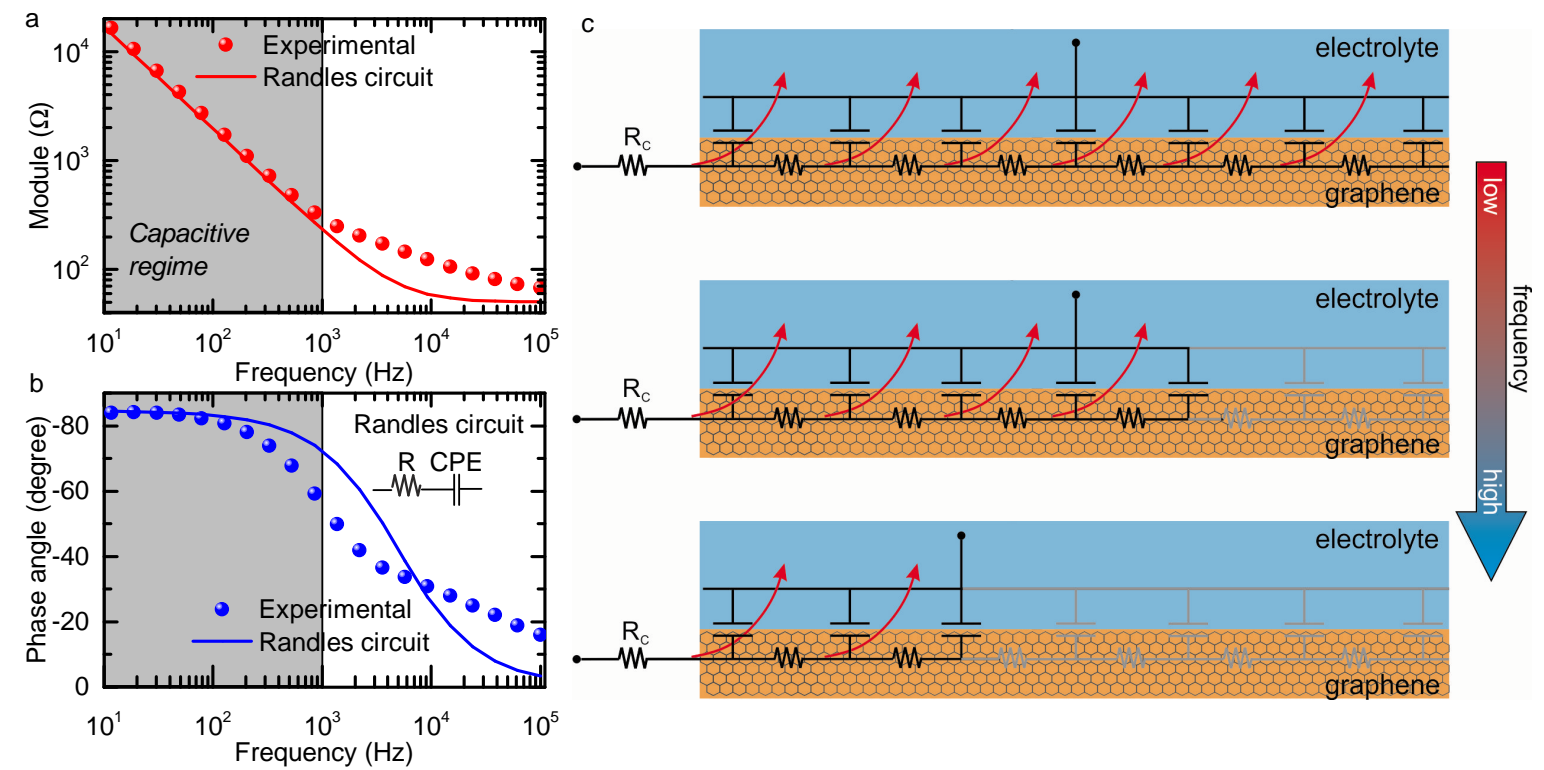

Figure 2. Illustration of the frequency response in graphene electrodes. Frequency dependence of (a) the module and (b) the phase angle of the measured impedance (symbols) of SLG in an electrode configuration. The solid lines represent the fit using a Randles circuit consisting of a resistor and a CPE in series. The reason for the discrepancy between the fit and the data is illustrated in (c) where the equivalent circuit elements contributing to the current density are shown for different frequencies. The gray shaded area in (a) and (b) represents the capacitive regime and the white area corresponds to the frequencies where the influence of the distributed elements on the frequency response can be observed. In (c), $R_{c}$ corresponds to a series resistance in the circuit, representing the contribution of the contact resistance and the access resistance; the red lines represent the current path in the device.

Figure $2 \mathrm{a}$ and $\mathrm{b}$ show the Bode plot, i.e. the impedance and the phase angle of SLG measured in an electrode configuration (Fig. 1a left), for a DC potential near the charge neutrality point (solid symbols). At frequencies lower than approximately 1 
$\mathrm{kHz}$, an almost purely capacitive regime with a phase shift near $-90^{\circ}$ can be observed. At higher frequencies (1 to $100 \mathrm{kHz}$ ), the electrode shows a mixed response (capacitive and resistive) with a phase angle between $-45^{\circ}$ and $0^{\circ}$. A simple equivalent circuit model consisting of a resistor, representing the series combination of the contact, access, and electrolyte resistances, and a CPE (see inset in Fig. 2b) can be used to reproduce the data at low frequencies $(<100 \mathrm{kHz})$. At higher frequencies $(>100 \mathrm{kHz})$, however, this circuit is not sufficient to fit the measured impedance and a more complex modeling is required. As schematically explained in Fig. 2c, the graphene/electrolyte interface is governed by a distribution of elements, resistors and capacitors, along the graphene surface resulting from the limited conductivity of graphene. Charge carriers can flow through different paths along the graphene sheet before charging the distributed capacitor elements; the impedance of each current path corresponds to the impedance of the capacitive element plus the resistance of the graphene from the contact to the corresponding capacitive element. At low frequencies, the impedance of the current paths is dominated by the capacitive elements; thus, all paths contribute equally to the total current (Fig. 2c top). Increasing the frequency leads to a reduction of the impedance of the capacitance elements with respect to the resistive paths. As a result, the current paths do not contribute equally to the current (Fig. 2c center). The higher the frequency is, the more relevant this effect gets, and the current will preferentially flow through the paths that are very close to the contact (Fig. 2c bottom and Fig. S10). However, for graphene-related materials with a low sheet resistance, as it is the case of multilayer graphene (MLG), the influence of the distributed elements can be neglected and the impedance of the electrode can be modelled using a simple equivalent circuit consisting of a resistor and a CPE (see S.I.).

The frequency-dependent response of the distribution of elements in SLG can be modeled as follows. Considering a circular graphene sheet (Fig. 1a left) with $r$ being the radial distance to the center, the following differential equations can be written to correlate the measured current $I$ with the applied potential $U:[20]$

$$
\begin{aligned}
& \frac{d I(r, \omega)}{d r}=\frac{1}{Z_{i}(r, \omega)} U(r, \omega) \\
& I(r, \omega)=\frac{2 \pi r}{R_{n}} \frac{d U(r, \omega)}{d r}
\end{aligned}
$$

where $Z_{i}$ is the interfacial impedance represented by $C_{d}$ and $Z_{f}$ in Fig. 1c, and $\omega$ is the angular frequency. Solving these equations (see S.I.) yields for the total impedance $Z$ of the graphene electrode:

$$
Z(\omega)=\frac{-R_{n} J_{0}(b L, \omega)}{2 \pi L b J_{1}(b L, \omega)}
$$

with $L$ being the radius of the circular electrode and $J_{0}$ and $J_{1}$ the hyperbolic Bessel functions of the first kind and of the zeroth and first order, respectively. In the coefficient $b^{2}=-\frac{R_{n}}{2 \pi r Z_{i}(\omega)}, Z_{i}$ can be considered as the complex impedance of a constant phase element $(\mathrm{CPE}) Z_{i}(\omega)=\frac{1}{Q_{0}(i \omega)^{\beta}}$ with the parameters $Q_{0}$ and $\beta$ (see S.I.) and the 
imaginary number $i$, since Faradaic reactions (represented by $Z_{f}$ in Fig. 1c) can be excluded.

At high frequencies, Eq. 3 predicts that the real and imaginary parts are the same and thus the phase should reach a value of $-45^{\circ}[20]$, which is not observed experimentally (see Fig. 2b). The discrepancy is attributed to the contribution of the resistance $R_{c}$ in series with $Z$ (Fig. 2c). $R_{c}$ is the sum of contributions from the electrolyte resistance, the contact resistance, as well as the so-called access resistance of the device, i.e. the resistance of the region of graphene not exposed to the electrolyte. The relation between the electrode impedance $Z$ and $R_{c}$ will determine the response at high frequencies (see S.I. for a detailed discussion of the effect of $R_{c}$ ). In the case of a non-zero $R_{c}$ value the phase at high frequencies can reach $0^{\circ}$ because $R_{c}$ is purely real and $Z$ decreases with increasing frequency.

Eq. 3 modified with the series contribution of $R_{c}$ has been used to fit the impedance measured as a function of the electrolyte potential in an aqueous electrolyte; $R_{n}, R_{c}$, and the parameters of the $\operatorname{CPE}\left(Q_{0}\right.$ and $\left.\beta\right)$ were used as the fitting parameters. In the case of the electrolyte-gated graphene devices, the expected position of the charge neutrality point is at $-0.1 \mathrm{~V}$, which is the difference of the work function of graphene $(4.6 \mathrm{eV})$ and the $\mathrm{Ag} / \mathrm{AgCl}$ reference electrode $(4.7 \mathrm{eV})[23]$. However, as will be shown below, the charge neutrality point was found to be at $U_{D}=0.05 \mathrm{~V}$ vs $\mathrm{Ag} / \mathrm{AgCl}$ (see S.I.), which is generally attributed to the p-doping introduced by the polymer used during the transfer (see Methods Section). Fig. 3a and b shows the measured module and phase angle (symbols) of the impedance, respectively, near to $\left(U_{D}+0.05 \mathrm{~V}\right)$ and away from $\left(U_{D}+0.35 \mathrm{~V}\right)$ the charge neutrality point. In both cases, an almost ideal capacitive regime can be observed at frequencies lower than $1 \mathrm{kHz}$. However, at frequencies higher than $1 \mathrm{kHz}$, the frequency response departs from the one predicted by the Randles circuit depending on the applied electrolyte potential. At potentials away from the charge neutrality point (red symbols), where the sheet resistance is lower, the electrode impedance is more dominated by $R_{c}$, as the phase shift approaching $0^{\circ}$ at high frequency shows. Near the charge neutrality point, where the sheet resistance is higher, the contribution of the distributed elements discussed above becomes dominant in the high frequency regime. The solid lines represent the fit according to Eq. 3 modified with the series contribution of $R_{c}$ showing an excellent agreement with the measured data. The dashed line in Fig. 3b represents the case $R_{c}=0$. A fit using the finite element method (FEM) is presented in the S.I.. Fig. 3c depicts the capacitance of the SLG electrode (green symbols) as a function of the gate voltage $U_{G}$ with respect to the charge neutrality point voltage $U_{D}$. Here, the capacitance has been obtained from the fitted $Q_{0}$ parameter of CPE and the parameter $\beta$ was found to be 0.96 . The experimental data is in good agreement with a calculated capacitance (orange line) that takes into account the series combination of the electric double layer capacitance and the quantum capacitance (purple line), calculated using an extended Poisson-Boltzmann model[21, 22]. The lowest measured capacitance is $1.15 \mu \mathrm{F} \mathrm{cm}^{-2}$, which is slightly higher than the calculated value. We tentatively attribute this discrepancy to the contribution 
of impurities in the graphene sheet like defects and doping, which are known to be introduced during the graphene transfer[24] (see Methods Section and S.I.). Away from the charge neutrality point, the capacitance increases and starts to saturate at values above $2.0 \mu \mathrm{F} \mathrm{cm}^{-2}$, which has been previously discussed in terms of the dominant contribution of the electrical double layer capacitance over the quantum capacitance[13]. The intrinsic sheet resistance, extracted from fitting the impedance spectra at different electrolyte voltages, has its maximum $(4 \mathrm{k} \Omega)$ at the charge neutrality point $(0.05 \mathrm{~V}$ vs $\mathrm{Ag} / \mathrm{AgCl}$ ) and decreases away from the charge neutrality point (see Fig. 3d). At very high frequencies $(>100 \mathrm{kHz})$, the module of the graphene electrode impedance approaches asymptotically a resistive term which represents the combined contribution
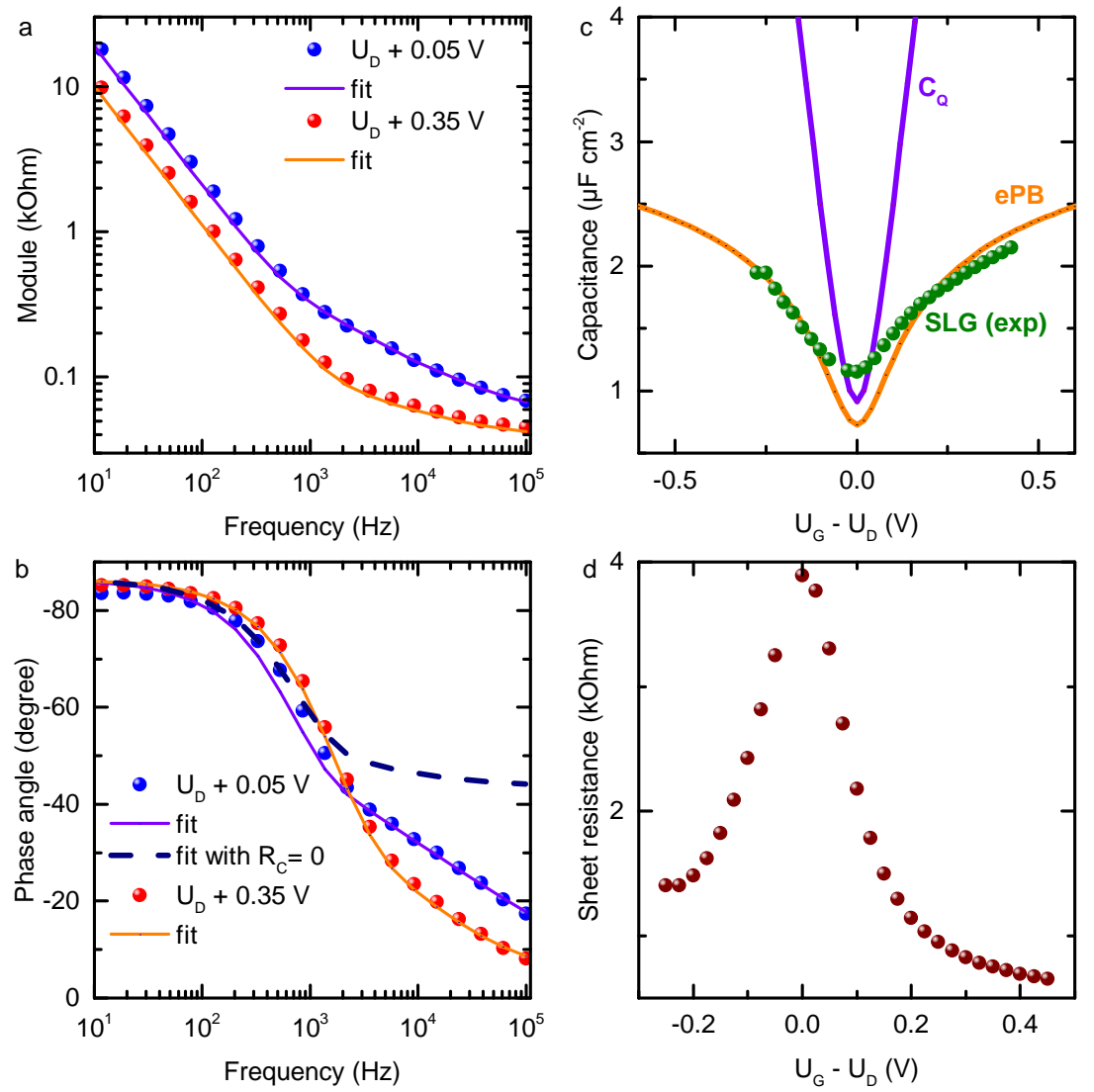

Figure 3. Impedance of graphene electrodes. Module (a) and phase angle (b) of SLG (symbols) measured in electrode configuration for two exemplary gate potentials close to $\left(U_{D}+0.05 \mathrm{~V}\right)$ and away from $\left(U_{D}+0.35 \mathrm{~V}\right)$ the charge neutrality point, and fitted (solid lines) using the analytical solution of the distribution of elements. The dashed line in (b) simulates the expected phase in the case $R_{c}=0$. (c) CPE capacitance $\left(Q_{0}\right)$ of SLG (symbols) as a function of the gate voltage $U_{G}$ with respect to the charge neutrality point voltage $U_{D}$; for comparison, the calculated capacitance (orange line), using an extended Poisson-Boltzmann model[21, 22] that takes the quantum capacitance $C_{Q}$ and the double layer capacitance into account, is also shown. (d) Calculated intrinsic sheet resistance as a function of the applied potential. 
Frequency Response of Electrolyte-gated Graphene Electrodes and Transistors

of the contact, access, and electrolyte resistance.
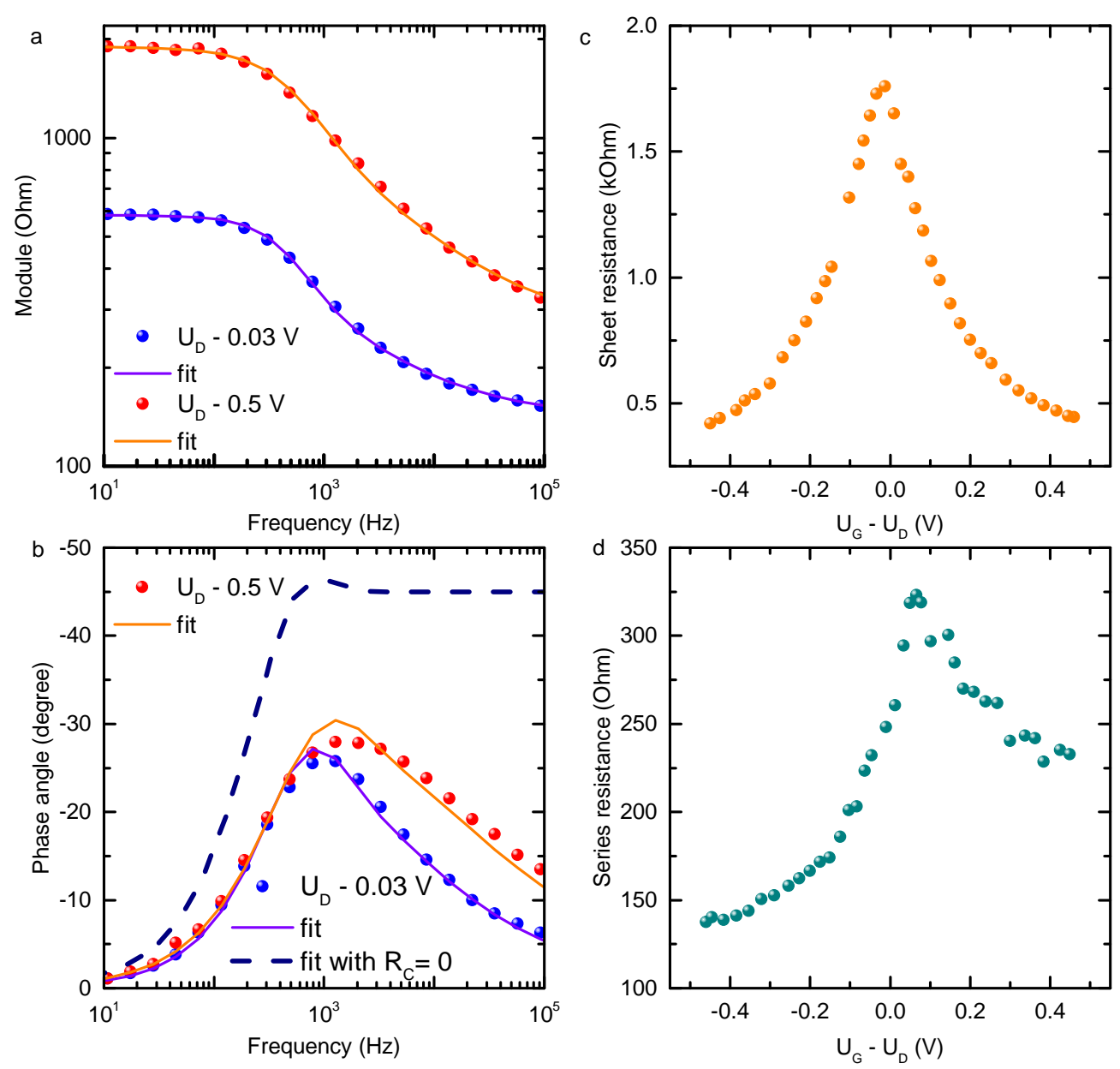

e
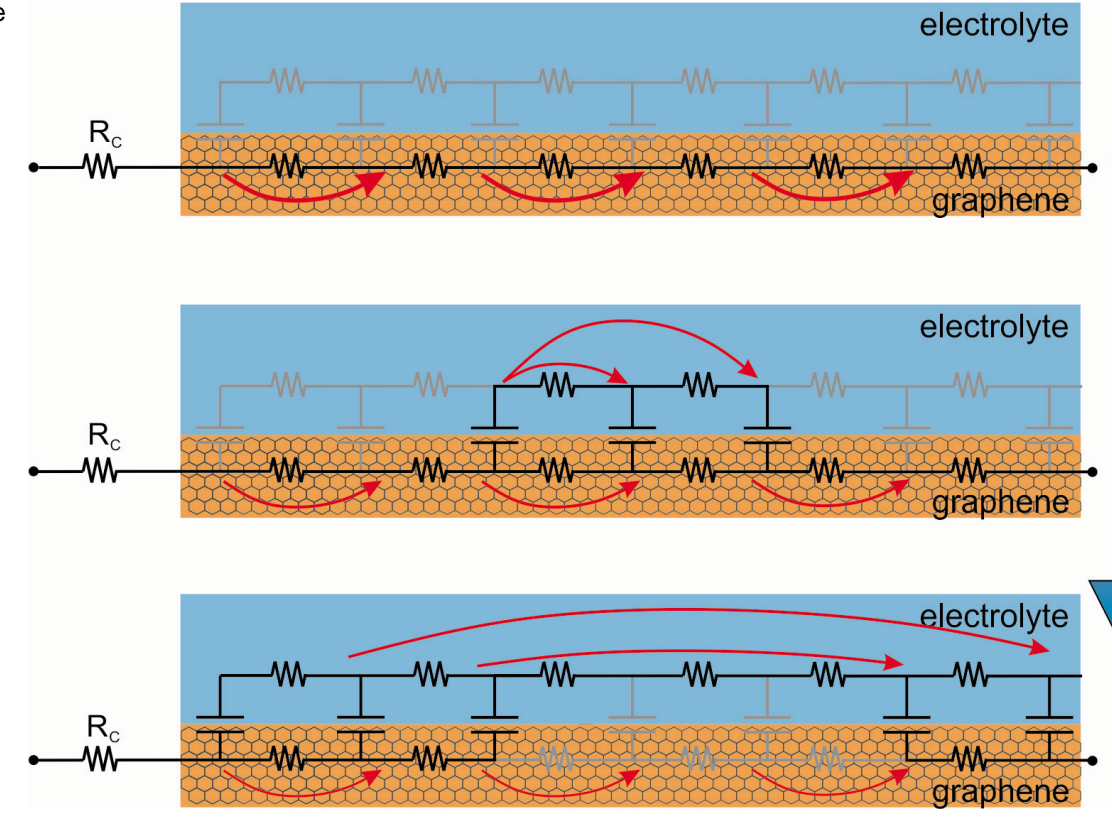
Figure 4. Impedance of graphene transistors. Module (a) and phase angle (b) of SLG measured in a transistor configuration (symbols) for two different gate potentials. The solid lines represent the fits according to the analytical solution of the distribution of elements. In part (b), the dashed line corresponds to a simulation in which $R_{c}=0$. (c) Extracted intrinsic and (d) series resistance as a function of the applied gate voltage $U_{G}$ using the analytical model of the distribution of elements. (e) Illustration of the circuit elements contributing to the current density at the graphene/electrolyte interface at different frequencies.

Similar to the case of the electrode configuration, the distribution of elements has a great influence on the frequency response of SLG electrolyte-gated field-effect transistors. Fig. 4 shows the impedance of SLG measured in a transistor configuration (Fig. 1a (right)), i.e. it represents the impedance measured between the drain and the source contacts. To this end, an AC voltage was applied between drain and source while an external voltage $U_{G}$ was applied to the electrolyte to change the position of the Fermi level in graphene. At high frequencies $(\approx 100 \mathrm{kHz})$, the transistor shows a non-resistive behavior with a phase angle significantly more negative than $0^{\circ}$ for gate bias near $\left(U_{D}-0.03 \mathrm{~V}\right.$, blue symbols) and away $\left(U_{D}-0.5 \mathrm{~V}\right.$, red symbols) from the charge neutrality point (Fig. 4b). With decreasing frequency, the phase angle reaches a minimum close to $-30^{\circ}$ at around $2 \mathrm{kHz}$, and increases to $0^{\circ}$ at frequencies lower than $10 \mathrm{~Hz}$. Accordingly, the module of the impedance (Fig. 4a) does not exhibit any frequency dependence at frequencies below $10 \mathrm{~Hz}$; in this frequency regime, the measured impedance corresponds to the resistance of the graphene sheet from source to drain. As expected, the graphene sheet resistance depends on the applied gate voltage and varies from $1.8 \mathrm{k} \Omega$ near the charge neutrality point to $0.42 \mathrm{k} \Omega$ away from the charge neutrality point (Fig. 4c). At low frequencies (below $10 \mathrm{~Hz}$ for the measured device), the impedance of the distributed capacitive elements representing the graphene/electrolyte interface is significantly larger than the impedance of the resistive elements representing the graphene sheet resistance; as a result, it can be assumed that the only equivalent circuit elements contributing to the current density are the resistors along the graphene sheet (see illustration in Fig. 4e and Fig. S13). With increasing frequency, however, the impedance of the interfacial capacitive elements decreases and its contribution cannot be neglected anymore. As a result, part of the injected signal at the drain or source contact "leaks" through the graphene/electrolyte interface, which offers an alternative conduction path through the capacitors and the electrolyte. This leads to a decrease in the phase angle towards values close to $-45^{\circ}$ at high frequencies, characteristic of an interface with resistive and capacitive distributed elements as shown in the illustration of Fig 4e. At high frequencies ( $\geq 2 \mathrm{kHz}$ ), the phase angle of the measured impedance approaches again $0^{\circ}$ : in this frequency range, the flow of charge from source to drain across the electrolyte (low impedance path due to the used high ionic strength) becomes dominant over the flow along the graphene sheet. As a result, the frequency response is dominated by the series resistance $R_{c}$, as already discussed for the case of the electrode configuration (a detailed discussion of the contribution of $R_{c}$ in the transistor configuration is provided in the S.I.). The analytical solution of the distribution of 
elements with a rectangular graphene area (the full derivation is presented in the S.I.) is used to fit the measured data and is represented by the solid lines in Fig. 4a and b. The dashed line represents the case in which $R_{c}=0$. The fits show a good agreement except for the frequency range around $2 \mathrm{kHz}$ where the maximum phase angle difference is observed. This deviation can be explained by oversimplifications of the analytical model like a negligible electrolyte resistance and a homogeneous graphene conductivity and capacitance along the channel. Simulations of the impedance using a FEM model that is able to overcome the limitations of the analytical solution can be found in the S.I.. An important outcome of this work is the realization that the discussed analytical model can be used to extract the series resistance (Fig. 4c), which includes the contact resistance, and the interfacial capacitance (Fig. S6) using the frequency-dependent drain-to-source impedance. The voltage dependence of the extracted series resistance results from the ambipolar transport in graphene and the characteristic p-type doping of CVD graphene[24]. When the transistor is biased in the hole regime $\left(U_{G}<U_{D}\right)$, the Fermi level in channel area is in the graphene valence band, resulting in the formation of a pp-junction at the interface between the channel and the ungateable p-doped graphene near the metal contacts. In the electron region $\left(U_{G}>U_{D}\right)$, in contrast, a pn-junction is formed which increases the contact resistance and thus the series resistance.

We can define a threshold frequency $f_{\text {th }}$ above which the frequency response of the devices impedance is governed by the contribution of the distribution of elements. For the particular case of the transistor, the threshold frequency separates the resistive and non-resistive response of a transistor. It can be shown (see S.I.) that $f_{t h}=\frac{1}{2 \pi R_{n} C_{i n t}}$, i.e. it depends on the capacitance and resistance of the active channel and thus on the device dimension and the electronic properties of the used material. For instance, the threshold frequency of the transistor discussed above, with a dimension of $0.96 \mathrm{x}$ $0.96 \mathrm{~cm}^{2}$ is close to $1 \mathrm{kHz}$. While for a microtransistor $\left(10 \mathrm{x} 20 \mu \mathrm{m}^{2}\right)$ the threshold frequency was measured above $10 \mathrm{MHz}$ (see Fig. S14), the threshold frequency for a mm-sized SLG transistor is expected to be in the higher $\mathrm{kHz}$ range. On the other hand, for a mm-sized transistor based on reduced graphene oxide, with a typical sheet resistance of $43 \mathrm{k} \Omega \cdot \mathrm{sq}[25]$ and interfacial capacitance of $283 \mu \mathrm{F} \mathrm{cm}^{-2}$ [26], the threshold frequency is expected in the range of $1 \mathrm{~Hz}$, suggesting that the operation frequency of sensors based on reduced graphene oxide should be restricted to frequencies lower than $1 \mathrm{~Hz}$ in the case of mm-sized devices.

\section{Conclusion}

In conclusion, we have investigated the frequency-dependent impedance of SLG electrolyte-gated field-effect transistors and electrodes. Our work shows that the frequency response of the graphene/electrolyte interface has to be described considering a distribution of resistive and capacitive elements along the graphene surface related to the graphene sheet resistance and the interfacial capacitance. Using an analytical solution to the problem of the distribution of elements, the frequency response of SLG 
electrodes and transistors in terms of module and phase angle could be reproduced and the intrinsic resistance, the series resistance, and the interfacial capacitance could be obtained from the measurements in the electrode and transistor configurations. Our model is able to predict a threshold frequency above which the device response is dominated by the distribution of elements. In the particular case of the transistors, the threshold frequency separates the resistive and non-resistive regimes of the drainsource impedance. At frequencies higher than the frequency threshold, the capacitive impedance is low, therefore, the electrolyte provides a less resistive path for the signal injected to the drain or source contacts, which effectively short-circuits the drainsource conductive path along the electrolyte. Our results are of relevance especially for applications using electrolyte-gated graphene devices, for instance biosensing and bioelectronics applications, in order to understand the frequency response of the devices and, even more, to estimate at which frequency these devices can be operated.

\section{Methods}

\section{Fabrication of graphene electrodes and transistors}

Graphene was grown by chemical vapor deposition using copper as metal catalyst and methane as carbon source. Before growth, copper foil (25 $\mathrm{m}$ thickness, Alfa Aesar) was electropolished[27] for 5 minutes at a current density of $12 \mathrm{~mA} \mathrm{~cm}^{-2}$. After an annealing step at $1015^{\circ} \mathrm{C}$ with an argon flow of $400 \mathrm{sccm}$ and a hydrogen flow of $100 \mathrm{sccm}$ at 100 mbar, a methane flow of $0.2 \mathrm{sccm}$ for 30 minutes was introduced to start the graphene nucleation and growth at 15 mbar. In order to get full graphene coverage, the carbon supply was continuously increased by increasing the methane flow to $0.5 \mathrm{sccm}$. The copper was etched by in a chemical solution containing $0.5 \mathrm{M} \mathrm{FeCl}_{3}$ and $2 \mathrm{M} \mathrm{HCl}$ using PMMA 950K A2 as supportive layer. The graphene sheet was fished onto a glass substrate, which was cleaned in oxygen plasma (200 W, 3 minutes), and dried on a hotplate at $50{ }^{\circ} \mathrm{C}$. The polymer was removed in hot acetone for 1 hour. An aluminum wire was connected to the graphene sheet via silver paste and insulated with a silicone layer (Scrintec 601).

\section{Electrochemical Measurements}

All electrochemical measurements were recorded using a Parstat (Princeton Applied Technologies) system in a three electrode configuration with a $\mathrm{Ag} / \mathrm{AgCl}$ electrode as reference. In the electrode configuration, a platinum wire was used as counter electrode and the electrolyte consisted of $5 \mathrm{~mm}$ PBS buffer containing $500 \mathrm{~mm} \mathrm{KCl}$. The pH value was adjusted to $\mathrm{pH}=3$ by adding $\mathrm{HCl}$. In the transistor configuration, the gate potential has been changed using an external voltage source (Keithley 2400) in series with a $50 \mathrm{M} \Omega$ resistor. 


\section{Funding Sources}

This work has been partially supported by the German Research Foundation (DFG) in the framework of the Priority Program 1459 "Graphene", the Nanosystems Initiative Munich (NIM), and the European Union under the Graphene Flagship (Contract No. 604391). This work also received funding from the European Unions Horizon 2020 research and innovation programme under Grant Agreement No 696656 and from the Severo Ochoa Program (MINECO, Grant SEV-2013-0295).

\section{References}

[1] K. S. Novoselov, A. K. Geim, S. V. Morozov, D. Jiang, Y. Zhang, S. V. Dubonos, I. V. Grigorieva, and A. A. Firsov. Electric field effect in atomically thin carbon films. Science (New York, N.Y.), 306(5696):666-669, 2004.

[2] K. S. Novoselov, V. I. Fal'ko, L. Colombo, P. R. Gellert, M. G. Schwab, and K. Kim. A roadmap for graphene. Nature, 490(7419):192-200, 2012.

[3] K. I. Bolotin, K. J. Sikes, Z. Jiang, M. Klima, G. Fudenberg, J. Hone, P. Kim, and H. L. Stormer. Ultrahigh electron mobility in suspended graphene. Solid State Communications, 146(9-10):351$355,2008$.

[4] Haiqun Chen, Marc B. Müller, Kerry J. Gilmore, Gordon G. Wallace, and Dan Li. Mechanically Strong, Electrically Conductive, and Biocompatible Graphene Paper. Advanced Materials, 20(18):3557-3561, 2008.

[5] Yong-Jin Kim, Yuna Kim, Konstantin Novoselov, and Byung Hee Hong. Engineering electrical properties of graphene: Chemical approaches. 2D Materials, 2(4):042001, 2015.

[6] Mar Garcia-Hernandez and Jonathan Coleman. Materials science of graphene: A flagship perspective. 2D Materials, 3(1):010401, 2016.

[7] L. H. Hess, M. V. Hauf, M. Seifert, F. Speck, T. Seyller, M. Stutzmann, I. D. Sharp, and J. A. Garrido. High-transconductance graphene solution-gated field effect transistors. Applied Physics Letters, 99(3):033503, 2011.

[8] Tapas Kuila, Saswata Bose, Partha Khanra, Ananta Kumar Mishra, Nam Hoon Kim, and Joong Hee Lee. Recent advances in graphene-based biosensors. Biosensors and Bioelectronics, 26(12):4637-4648, 2011.

[9] Berit Koerbitzer, Peter Krauss, Christoph Nick, Sandeep Yadav, Joerg J. Schneider, and Christiane Thielemann. Graphene electrodes for stimulation of neuronal cells. 2D Materials, 3(2):024004, 2016.

[10] François Béguin, Volker Presser, Andrea Balducci, and Elzbieta Frackowiak. Carbons and Electrolytes for Advanced Supercapacitors. Advanced Materials, 26(14):2219-2251, 2014.

[11] Thangavelu Palaniselvam and Jong-Beom Baek. Graphene based 2D-materials for supercapacitors. 2D Materials, 2(3):032002, 2015.

[12] Yuanbo Zhang, Yan-Wen Tan, Horst L. Stormer, and Philip Kim. Experimental observation of the quantum Hall effect and Berry's phase in graphene. Nature, 438(7065):201-204, 2005.

[13] Jilin Xia, Fang Chen, Jinghong Li, and Nongjian Tao. Measurement of the quantum capacitance of graphene. Nature nanotechnology, 4(8):505-509, 2009.

[14] Mark E. Orazem and Bernard Tribollet. Electrochemical Impedance Spectroscopy. John Wiley \& Sons, Inc, Hoboken, NJ, USA, 2008.

[15] Chang-Hyun Kim and C. Daniel Frisbie. Determination of Quantum Capacitance and Band Filling Potential in Graphene Transistors with Dual Electrochemical and Field-Effect Gates. The Journal of Physical Chemistry C, 118(36):21160-21169, 2014.

[16] Hengxing Ji, Xin Zhao, Zhenhua Qiao, Jeil Jung, Yanwu Zhu, Yalin Lu, Li Li Zhang, Allan H. 
MacDonald, and Rodney S. Ruoff. Capacitance of carbon-based electrical double-layer capacitors. Nature communications, 5:3317, 2014.

[17] Jin-Hui Zhong, Jun-Yang Liu, Qiongyu Li, Mian-Gang Li, Zhi-Cong Zeng, Shu Hu, De-Yin $\mathrm{Wu}$, Weiwei Cai, and Bin Ren. Interfacial capacitance of graphene: Correlated differential capacitance and in situ electrochemical Raman spectroscopy study. Electrochimica Acta, 110:754-761, 2013.

[18] Meryl D. Stoller, Carl W. Magnuson, Yanwu Zhu, Shanthi Murali, Ji Won Suk, Richard Piner, and Rodney S. Ruoff. Interfacial capacitance of single layer graphene. Energy $\&$ Environmental Science, 4(11):4685, 2011.

[19] Benno M. Blaschke, Martin Lottner, Simon Drieschner, Andrea Bonaccini Calia, Karolina Stoiber, Lionel Rousseau, Gaëlle Lissourges, and Jose A. Garrido. Flexible graphene transistors for recording cell action potentials. 2D Materials, 3(2):025007, 2016.

[20] J. D. Wiley. C-V profiling of GaAs FET films. IEEE Transactions on Electron Devices, 25(11):1317-1324, 1978.

[21] Markus Dankerl, Moritz V. Hauf, Andreas Lippert, Lucas H. Hess, Stefan Birner, Ian D. Sharp, Ather Mahmood, Pierre Mallet, Jean-Yves Veuillen, Martin Stutzmann, and Jose A. Garrido. Graphene Solution-Gated Field-Effect Transistor Array for Sensing Applications. Advanced Functional Materials, 20(18):3117-3124, 2010.

[22] Nadine Schwierz, Dominik Horinek, and Roland R. Netz. Reversed Anionic Hofmeister Series: The Interplay of Surface Charge and Surface Polarity. Langmuir, 26(10):7370-7379, 2010.

[23] Seung Min Song, Jong Kyung Park, One Jae Sul, and Byung Jin Cho. Determination of work function of graphene under a metal electrode and its role in contact resistance. Nano letters, 12(8):3887-3892, 2012.

[24] Ji Won Suk, Wi Hyoung Lee, Jongho Lee, Harry Chou, Richard D. Piner, Yufeng Hao, Deji Akinwande, and Rodney S. Ruoff. Enhancement of the electrical properties of graphene grown by chemical vapor deposition via controlling the effects of polymer residue. Nano letters, 13(4):1462-1467, 2013.

[25] Goki Eda, Giovanni Fanchini, and Manish Chhowalla. Large-area ultrathin films of reduced graphene oxide as a transparent and flexible electronic material. Nature nanotechnology, 3(5):270-274, 2008.

[26] Kaixuan Sheng, Yiqing Sun, Chun Li, Wenjing Yuan, and Gaoquan Shi. Ultrahigh-rate supercapacitors based on eletrochemically reduced graphene oxide for ac line-filtering. Scientific reports, 2:247, 2012.

[27] Bin Zhang, Wi Hyoung Lee, Richard Piner, Iskandar Kholmanov, Yaping Wu, Huifeng Li, Hengxing Ji, and Rodney S. Ruoff. Low-Temperature Chemical Vapor Deposition Growth of Graphene from Toluene on Electropolished Copper Foils. ACS Nano, 6(3):2471-2476, 2012. 